\title{
156d. Bilanzierte Untersuchungen über die Verwertbarkeit einer neuen Aminosäurenlösung
}

\author{
A. Palatsik-Malente
}

\section{Balance Investigations on the Suitability of a New Amino-Acid Solution}

Summary. On the basis of clinical studies in 20 patients who had intravenous infusions of amino-acid solutions on a total of 120 trial days, the utilisation of the so-called potato-egg principle, as realised in the solution Aminosteril $\mathrm{KE} 800$, is regarded as a specially suitable method of supplying the human organism with specific nitrogen components. The patients, all adults suffering from extrapulmonary tuberculosis, received only $40 \mathrm{~g}$ margarine and $150 \mathrm{~g}$ special protein-poor bread (total 1600 cal per day) apart from an infusion of $1000 \mathrm{ml}$ of the above-named solution for a period of 6 days. Balance examinations of the $24 \mathrm{~h}$ urine in a doubleblind trial generally showed a completely clear nitrogen balance.

Zusammentassung. Auf Grund von klinischen Studien an 20 Patienten, denen an insgesamt 120 Versuchstagen i.v. Aminosäurelösungen infundiert wurden, wird die Ausnutzung des sog. Kartoffel-Ei-Prinzips, wie es in der Lösung Aminosteril K. E. 800 verwirklicht worden ist, als ein besonders günstiges Verfahren zur Versorgung des menschlichen Organismus mit spezifischen Stickstoffbausteinen angesehen. Die Patienten, ausschließlich an extrapulmonalen Tuberkulosen erkrankte Erwachsene, erhielten neben der Infusion von $1000 \mathrm{ml}$ der ebengenannten Lösung über 6 Tage hinweg nur $40 \mathrm{~g}$ Margarine und $150 \mathrm{~g}$ eines eiweißarmen Spezialbrotes (insgesamt 1600 Calorien pro Tag). Bilanzuntersuchungen im doppelten Blindversuch aus dem 24 Std-Urin ergaben im allgemeinen eine völlig ausgeglichene Stickstoffbilanz.

\section{Zusammenfassender Bericht über die Forums-Diskussion}

\section{Diskussionsleiter: K. Hutschenreuter-Homburg}

Auf die Frage, welche therapeutischen Konsequenzen sich aus den beim Schock möglicherweise auftretenden Mikrozirkulationsstörungen ergeben, wird von Lawin, Hamburg, die rechtzeitige Heparingabe vorgeschlagen. Lawin vertritt auch die Auffassung, daß sich die Folgen einer Verbrauchskoagulopathie besonders an den Lungen manifestieren und deshalb das Problem eines durch Trauma zentral ausgelösten Lungenödems umstritten sei.

Als Frühsymptom der Verbrauchskoagulopathie wird nach Popov, Bonn, nicht nur ein Abfall der Thrombocytenzahl, sondern insbesondere eine Thrombocytenfunktionsstörung angesehen. Freilich muß eingeräumt werden, daß es noch nicht; allen Krankenhäusern jederzeit möglich sein dürfte, einen mehr oder minder aufwendigen Gerinnungsstatus zu erheben. Nach Meinung von Popov ist es aber ausreichend, wenn vor allem die Thrombocytenfunktion in den ersten Tagen täglieh einmal und dann später nur noch wöchentlich zweimal kontrolliert wird.

Treten unter einer Heparinbehandlung Blutungen auf (z. B. aus Wunden oder aus dem Gastrointestinaltrakt) hält Dohmen, Bonn, die sofortige Unterbrechung der Heparintherapie für unbedingt angezeigt. 